\title{
Feasibility of optoacoustic visualization of high-intensity focused ultrasound-induced thermal lesions in live tissue
}

\section{Parag V. Chitnis}

F. L. Lizzi Center for Biomedical Engineering Riverside Research Institute

156 William Street, 9th Floor

New York, New York 10038

\author{
Hans-Peter Brecht \\ Richard Su \\ Alexander A. Oraevsky \\ Fairway Medical Technologies \\ 710 N. Post Oak Road, Suite 204 \\ Houston, Texas 77024
}

\begin{abstract}
A 3-D optoacoustic imaging system was used to visualize thermal lesions produced in vivo using high-intensity focused ultrasound (HIFU). A 7.5-MHz, surgical, focused transducer with a radius of curvature of $35 \mathrm{~mm}$ and an aperture diameter of $23 \mathrm{~mm}$ was used to generate HIFU. A pulsed laser, which could operate at $755 \mathrm{~nm}$ and $1064 \mathrm{~nm}$, was used to illuminate excised tissue and mice using a bifurcated fiber bundle resulting in two wide beams of light. Tomographic images were obtained while the specimens were rotated within a sphere outlined by a concave arc-shaped array of 64 piezocomposite transducers. These images were then combined to reconstruct 3-D volume images (voxel resolution $0.5 \mathrm{~mm}$ ), which were acquired before and after HIFU exposure. In vivo optoacoustic images acquired at $1064 \mathrm{~nm}$ provided visualization of HIFU lesions. The lesion was indicated by a negative optoacoustic contrast. The molecular nature of such contrast may possibly be associated with reduction of the optical absorption due to reduced concentration of blood, tissue dehydration, denaturation of proteins and porphyrins, and reduction of thermoacoustic efficiency in the thermally treated tissue. These preliminary results demonstrate the potential of optoacoustic imaging to assess and monitor the progress of HIFU therapy. () 2010 Society of PhotoOptical Instrumentation Engineers. [DOI: 10.1117/1.3339977]
\end{abstract}

Keywords: optoacoustic; imaging; high-intensity focused ultrasound.

Paper 09366SSRR received Aug. 17, 2009; revised manuscript received Dec. 28 , 2009; accepted for publication Dec. 28, 2009; published online Mar. 11, 2010.

\section{Introduction}

High-intensity focused ultrasound (HIFU) provides a noninvasive and nonionizing alternative for treating tumors without damaging the surrounding healthy tissue. In HIFU therapy, absorption of ultrasonic energy at the focus leads to local heating that can result in apoptosis and thermal necrosis when the local temperature rises above $60{ }^{\circ} \mathrm{C}$ (Ref. 1). However, success of this therapeutic modality depends critically on the ability to focus HIFU energy accurately at the diseased site and assess the progress of the treatment in real time. ${ }^{2}$ Mainstream research efforts to guide and monitor HIFU therapy are concentrated on image-guided solutions based on either magnetic-resonance imaging, ${ }^{3}$ which is the current gold standard but cost prohibitive, or ultrasound imaging, ${ }^{4,5}$ which is economical but lacks precision and accuracy and often involves complex signal processing and model-based schemes.

In this letter, a study investigating the feasibility of monitoring HIFU using optoacoustic (OA) imaging, also referred to as photoacoustic (PA) imaging, is described. OA imaging is an emerging medical imaging technology that provides highresolution images of soft tissues based on optical contrast. ${ }^{6,7}$

Address all correspondence to: Parag V. Chitnis, F. L. Lizzi Center for Biomedical Engineering Riverside Research Institute, 156 William Street, 9th Floor, New York, NY 10038. Tel: 212-502-1749; Fax: 212-502-1729; E-mail: pchitnis@rrinyc.org
One clinical application that was recently demonstrated is the diagnostic imaging of breast cancer. ${ }^{8}$ Another potential application of OA imaging that has been demonstrated only in vitro before now is monitoring of thermally induced changes in tissue properties. ${ }^{9}$ Because OA imaging combines molecular selectivity of optical spectroscopy with the spatial resolution and real-time monitoring capability of ultrasound, it enables one to image both cancerous tumors ${ }^{8}$ and the formation of thermal lesions with adequate contrast relative to the surrounding tissue. ${ }^{10-12}$

OA imaging relies on the local absorption of a short laser pulse in the medium followed by a thermoacoustic transformation of the optical energy, resulting in the generation of ultra-wideband ultrasonic waves, which can be detected and used to generate an OA image. ${ }^{13} \mathrm{~A}$ review of the OA imaging principles, applications, and latest accomplishments, has been recently published in Ref. 14. The OA pressure $p$ is described by the following equation:

$$
\Delta p-\frac{1}{c_{0}^{2}} \frac{\partial^{2} p}{\partial t^{2}}=-\frac{\beta \mu_{a}}{C_{p}} \frac{\partial \phi}{\partial t},
$$

where $c_{0}$ is the adiabatic sound speed, $\beta$ is the isobaric thermal expansion coefficient, $C_{p}$ is the specific heat capacity of

1083-3668/2010/15(2)/021313/5/\$25.00 @ 2010 SPIE 


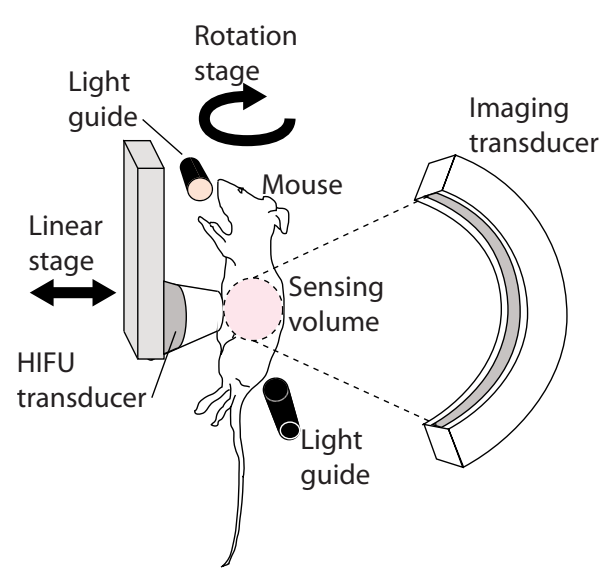

Fig. 1 Schematic diagram of the experimental setup. The specimen was rotated and tomographic slices of the OA scans were acquired incrementally before and after HIFU exposures. The HIFU transducer was moved away from the specimen during imaging.

tissue, $\mu_{a}$ is the absorption coefficient, and $\phi$ is the optical fluence.

OA imaging can provide anatomical mapping of the tumor and its surrounding vasculature along with functional information about blood concentration and its level of oxygenation. ${ }^{15,16}$ Although OA imaging has been used to provide in vivo images of blood vessels, organs, and tumors, ${ }^{17-20}$ it has not been used to visualize or monitor HIFU-induced lesions in vivo. However, in vitro studies have demonstrated the possibility of optoacoustically monitoring HIFU therapy by demonstrating OA-based imaging of the thermal lesions in tissue, ${ }^{10}$ and further by characterizing the optical extinction coefficient and the corresponding OA profile at $1064 \mathrm{~nm}$ for both raw and thermally necrotic liver. ${ }^{11}$ This study was designed to test the capability of OA imaging in monitoring changes of tissue OA properties induced by HIFU in vivo, and to compare the data with results previously obtained in vitro. We hypothesized that the HIFU-induced thermal lesion will exhibit a change in the OA response likely related to a change in the local concentration of the optically absorbing chromophores. However, we could not exclude the possibility of thermally induced changes in the thermoacoustic efficiency, which includes variations in the thermal expansion coefficient and the speed of sound in tissue. The proposed method was developed to exploit the functional information provided by this modality to image and distinguish between concentration of optical absorbers in the HIFU-induced lesion and the surrounding living tissue.

\section{Materials and Methods}

Studies were conducted on excised beef and chicken muscles as well as live nude mice using the 3-D OA imaging system described in Ref. 21. Briefly, the imaging system consisted of three main components: light delivery, tissue/animal translation and rotation, and the detector array and its associated electronics for data acquisition and imaging reconstruction. A representative schematic diagram of the experimental setup is shown in Fig. 1.

The OA images were acquired at two wavelengths, $755 \mathrm{~nm}$ and $1064 \mathrm{~nm}$, using a pulsed laser (Quanta Systems,
Solbiate Olona VA, Italy). The laser emitted $100 \mathrm{~mJ}$ per pulse (at both wavelengths) into the input of the fiber bundle. Light delivery was performed orthogonally to the array of acoustic transducers (described in the following) by a bifurcated, randomized fiber bundle (Schott AG, Mainz, Germany) of 48 inches in length. After leaving the fiber, the two light beams, $E=25 \mathrm{~mJ}$ each, were expanded to a diameter of $8 \mathrm{~cm}$ at the target.

The OA signals were acquired with a custom-designed, 64-element, curved ultrasonic array with a specified center frequency of $3.1 \mathrm{MHz}$ (Imasonic SAS, Voray sur l'Ognon, France) while the specimen was rotated incrementally. The $(-6 \mathrm{~dB})$ bandwidth of the array was $80 \%$. The piezocomposite elements were $2 \mathrm{~mm} \times 2 \mathrm{~mm}$ and were spaced $0.7 \mathrm{~mm}$ apart. The focal length of the array was $65 \mathrm{~mm}$, and the overall angle between element 1 and 64 was $152 \mathrm{deg}$, which gave an angle of $2.4 \mathrm{deg}$ between elements. The step size for the rotation was selected at $2.4 \mathrm{deg}$ to ensure even detector spacing in equatorial and meridian directions as well as cubic voxel dimensions. The present methods of signal and image processing permitted qualitative differentiation of tissues with different optical absorption, which in turn relates to relative differences in optically absorbing chromophores, such as hemoglobin of blood and water. The 3-D tomography system was capable of generating images with a spatial resolution of $0.5 \mathrm{~mm}$, with equal axial (depth) and lateral resolution. Images were acquired before and after administering HIFU to tissue specimens and mice. During the imaging, the HIFU transducer was moved away from the specimen using a manual-linear stage to allow rotation of the specimen.

A surgical HIFU transducer (Model SU-109, Sonic Concepts, Inc., Bothell, Washington) was used to produce thermal lesions. The transducer had a nominal center frequency of $7.5 \mathrm{MHz}$, a radius of curvature of $35 \mathrm{~mm}$, and an aperture diameter of $23 \mathrm{~mm}$. The transducer was driven in continuouswave mode using a function generator (Model 3314A, Hewlett Packard, Santa Clara, California) and an RF power amplifier (Model 2100L, Electronic Navigation Industries, Rochester, New York) with a 50-dB gain. All specimens were subjected to $30 \mathrm{~s}$ of HIFU exposure. The focal point acoustic intensity measured in degassed water using a radiation force balance was $5.5 \mathrm{~kW} / \mathrm{cm}^{2}$.

In vivo studies were performed as per NIH guidelines for animal use, and a total of four mice were used. The mouse was anesthetized with $1.5 \%$ isoflourane in a mixture of pure oxygen and room air (50:50 ratio) using an isoflourane distribution unit (Summit Anesthesia Solutions, Bend, Oregon) and then placed into a custom-made mouse holder. The anesthesia gas was delivered to the mouse holder via a central freely rotating shaft. The head of the mouse was placed in a cone while the anesthesia gas generated a diving bell and allowed the mouse to freely breathe while placed in the tank under water with excess gas bubbling into the tank. The mice were subjected to HIFU exposures at various locations in the abdominal section in an attempt to produce a lesion on the kidneys and the liver. Euthanasia was performed after the experiment through spinal dislocation under 5\% isoflourane in oxygen and organs were dissected for visual inspection.

Experiments were performed in a custom-made water tank $\left(50.8 \mathrm{~L} \times 41 \mathrm{~W} \times 43 \mathrm{H} \mathrm{cm}^{3}\right)$. The temperature in the water 
tank was maintained at $36{ }^{\circ} \mathrm{C}$ by heating elements placed underneath. The rotation of the mouse was performed by a DC motor with an optical encoder (Faulhaber Gmbh, Schoenaich, Germany), while the position of the array was controlled by three motorized linear stages (IAI, Inc., Torrance, California).

\section{Results}

We acquired OA images of two different tissue samples in vitro, bovine muscle and chicken breast, using two laser wavelengths, $755 \mathrm{~nm}$ and $1064 \mathrm{~nm}$. Limitations of the HIFU transducer in its maximum power and the total time of HIFU emission resulted in generation of only mild thermal lesions. These lesions were not prominently visible to the naked eye on a slice of tissue, and the optical contrast between the thermally denatured region and the surrounding tissue was not adequate for OA visualization. A relative comparison between the two wavelengths indicated that OA imaging performed at $1064 \mathrm{~nm}$ gave a marginally better indication of a change in the local optical property but failed to clearly demarcate the thermal lesion. A more powerful HIFU transducer and a longer irradiation time could produce lesions that are more prominently visible and detectable with an OA imaging system, as previously reported. ${ }^{10-12}$ Lack of adequate contrast in the initial in vitro results motivated studies to investigate OAbased detection of thermal lesions in vivo using blood-rich tissue with greater background optical absorption, such as the kidney. Consequently, in vivo OA imaging of tissue with a high concentration of hemoglobin and water resulted in a stronger contrast (larger changes of the tissue optical properties) of a relatively mild thermal lesion.

In all in vivo experiments, the precise location of the HIFU transducer's focus was not known a priori. In two cases, misalignment of the HIFU transducer's focus combined with its limited power failed to produce a lesion in any of the major abdominal organs as determined upon dissection. In these cases, the OA images did not indicate the presence of any lesions. Figure 2 shows a representative result from the in vivo studies acquired using a pulsed $\mathrm{Nd}$ :YAG laser operated at $1064 \mathrm{~nm}$. OA images were acquired and converted into 3-D grayscale intensity maps before (a) and after (b) HIFU exposures. In this instance, a comparison between pre- and postHIFU images clearly indicated a region in the left kidney with a negative OA contrast. Upon dissection and visual inspection, a white lesion characteristic of thermal necrosis resulting from HIFU was clearly visible on the left kidney in the same region as indicated by the in vivo OA image. Furthermore, the diameter of the lesion on the surface of the dissected kidney was roughly $2 \mathrm{~mm}$, which is in the range of that estimated from OA slices along the HIFU focus (1.5 to $2 \mathrm{~mm}$ ).

\section{Discussion}

The parameter that defines severity of a thermal lesion is a product of power and time, i.e., the total deposited energy. Very aggressive deposition of energy in live tissues was shown to induce rapid dehydration and denaturation of proteins, resulting in tissue hardening and expulsion of blood from the treated volume (referred to as a hemorrhagic ring). ${ }^{22}$ However, this type of thermal therapy cannot be wellcontrolled, often resulting in undesirable side effects of char-

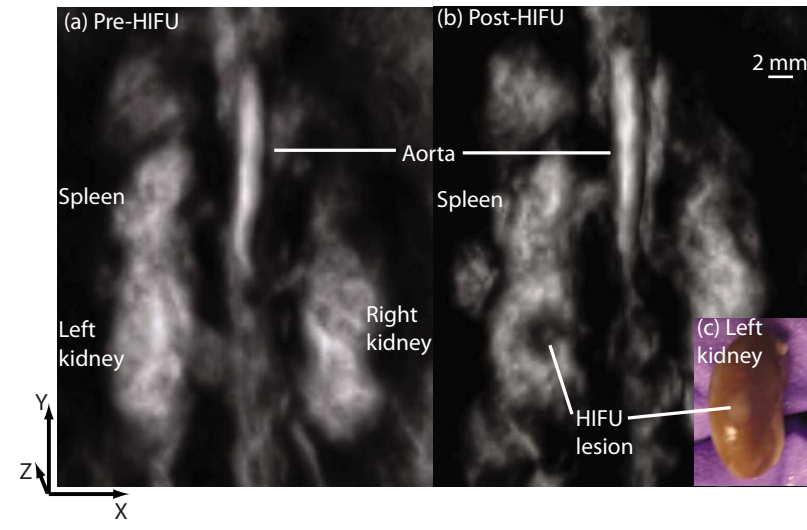

Fig. 2 Reconstructed OA images of the abdominal volume of a mouse using 1064-nm light acquired (a) pre-HIFU exposure and (b) postHIFU exposure. Grayscale intensity represents the magnitude of the local OA signal, which is related to the local optical absorption. The HIFU transducer's focus was aligned at the left kidney, and the HIFU axis was parallel to the $z$ axis. The post-HIFU image clearly shows a region in the left kidney with reduced optical contrast (lower blood concentration) corresponding to the HIFU-induced lesion as confirmed upon visual inspection (c).

ring and cavitation, and significant collateral damage. In contrast, HIFU therapy at moderate powers can produce thermal lesions with high precision and control. The HIFU lesion in our experiments was mild and soft, without any discernable hardening or hemorrhagic ring. Partially denatured protein lesions of this variety offer very little ultrasound contrast in lieu of acoustic backscatter. Therefore, detection and imaging of these mild lesions is often challenging using conventional imaging techniques such as B-mode diagnostic ultrasound. ${ }^{23} \mathrm{Be}-$ cause the OA imaging modality exploits the optical contrast associated with changes in the concentration of tissue chromophores, it provides a direct indication of modifications that occur in the molecular composition of denatured tissue, making this technique optimally suited for imaging HIFU lesions. Similar to the excised tissue results, the only in vivo images that resulted in unambiguous visualization of the HIFUinduced lesion were images acquired using the 1064-nm laser wavelength. In the OA images depicted in Fig. 2, gray scale represents the magnitude of the local OA signal, which relates directly to the locally absorbed optical energy. The two major tissue chromophores that absorb at the wavelength of $1064 \mathrm{~nm}$ are oxygenated hemoglobin of blood and water. Thus, the gray scale in the images likely relates to the local concentration of blood and water. Increased optical scattering of denatured tissue can change the effective local optical fluence, and in turn, can also result in an OA contrast. This scattering-based contrast, however, is likely insignificant in mild and soft thermal lesions compared to frequently reported, fully denatured and dehydrated tissues of hard thermal lesions.

The negative contrast observed in the thermal lesion as displayed in Fig. 2 is consistent with the hypothesis that the volume of tissue exposed to HIFU and subjected to thermal damage exhibits a reduction in the concentration of blood and water, molecules that strongly absorb the illumination at $1064 \mathrm{~nm}$. The stronger OA contrast at $1064 \mathrm{~nm}$ might be attributed to the optical absorption of (oxygenated) hemoglo- 
bin and water, both of which possess significantly higher optical absorption coefficients at $1064 \mathrm{~nm}$ as compared with $755 \mathrm{~nm}$. Thus, a stronger background optical absorption leads to a better sensitivity (signal-to-noise ratio) of the OA monitoring. Our in vivo finding of a greater OA contrast at $1064 \mathrm{~nm}$ compared with $755 \mathrm{~nm}$ is consistent with results of previously reported in vitro studies. ${ }^{10}$

On the other hand, the negative contrast apparent from HIFU lesions produced in vivo might be inconsistent with the changes in local optical absorption upon thermal damage, as reported by Khokhlova et al., ${ }^{11}$ who discovered a twofold increase in the experimentally inferred optical absorption coefficient as a result of in vitro boiling of a sample of excised porcine liver. Larin et al. ${ }^{12}$ observed a similar enhancement in the optical absorption once the local temperature in excised liver, myocardium, and prostate exceeded the temperature of coagulation (about $53{ }^{\circ} \mathrm{C}$ ).

The discrepancy between our observations and prior works might be attributed to several factors. First, thermal dynamics in live tissues is substantially different from that excised tissues due to blood perfusion. Thus, thermochemical molecular reactions can be completely different in the two cases. This difference is further compounded by the attenuation and aberration due to the interlaying skin, fat, and tissues when performing HIFU in an in vivo setting. Second, our method of heat delivery and the total thermal deposition were different from those employed in Refs. 11 and 12. In the majority of the results described by Khokhlova et al., ${ }^{11}$ they simulated the formation of necrotic tissue by immersing the tissue specimen in boiling water for $10 \mathrm{~min}$. In their study, the enhancement in optical absorption (and consequently the OA signal) was significantly more prominent in the tissue sample that was boiled compared to the tissue that was subjected to HIFU. Larin et al. ${ }^{12}$ employed a continuous-wave laser and conduction heating to induce thermal damage in excised liver, myocardium, and prostate samples. Similar to Khokhlova et al., their study involved excessive and rapid heating of the tissue, resulting in a visible hemorrhagic ring. In the present study, the treated region was exposed to HIFU for a duration of $30 \mathrm{~s}$. Postdissection examination of the HIFU-induced lesions revealed that the thermally denatured tissue was soft, without any discernable hardening or hemorrhagic ring, which indicated that HIFU-induced thermal deposition was mild, unlike boiling. Khokhlova et al. attribute the enhanced optical absorption to the thermally induced formation of methemoglobin, which has an optical absorption coefficient at $1064 \mathrm{~nm}$ several times higher than hemoglobin. HIFU-induced temperature elevations in the current study might not have been sufficient for inducing the formation of methemoglobin, but might have led to destruction of chemical bonds that absorb near-infrared light, tissue dehydration, and expulsion of blood from the volume of the thermal lesion. These effects resulted in a loss of optical absorption instead of an increased optical absorption. Further studies are necessary to elucidate the origin of the contrast between the healthy and thermally denatured tissue and to determine the optimal parameters for facilitating OAbased visualization of HIFU lesions.

The results of the present work demonstrate that OA imaging potentially can be employed to monitor and assess treatment (both the location and the size of the treated volume) for noninvasive ablation of tumors using HIFU. OA signals can also be employed for active guidance of HIFU procedures; time reversal of the OA signal emanating from the tumor using a HIFU transducer array can automatically focus HIFU energy back at the tumor site despite the presence of abberative tissue inhomogeneities in the acoustic path. ${ }^{24}$ The sensitivity of the OA monitoring can be enhanced by allowing bioconjugated gold nanoparticles, tuned to absorb an appropriate wavelength of light, to accumulate within the tumor via extravasation or specific targeting. ${ }^{25}$ The proposed technique is particularly attractive for treating breast cancer, where coupling of both light and ultrasound into the diseased tissue volume can be achieved readily. However, development of new transrectal and transesophageal probes to deliver light and ultrasound to different organs may allow implementation of OA-guided HIFU to treat tumors in other parts of the body. Because the OA imaging involves detection of ultrasonic waves, it can be readily integrated into existing diagnostic ultrasound imaging systems to provide co-registered multimodality images. ${ }^{26,27}$ Furthermore, since the amplitude of the OA signal depends on the local temperature ${ }^{28} \mathrm{OA}$ imaging can also provide a local temperature map similar to that obtained using magnetic-resonance thermometry and can be used to monitor local temperature elevation in real time. With proper deconvolution of distortions introduced to the OA signals upon detection by the ultrasonic transducers and the electronic system, one can obtain quantitative information on tissue optical properties. ${ }^{29}$

\section{Conclusion}

Our study demonstrated that OA imaging can be used for monitoring the formation of a thermal lesion in tissues of live animals. The combination of high sensitivity to the optical contrast and high spatial resolution makes OA imaging uniquely suitable for monitoring the effects of thermal therapy. Initial results demonstrated that even a mild thermal lesion can be visualized and motivate further studies. An important future investigation would involve experiments to better understand the molecular nature of thermal processes that result in changes of tissue optical properties. OA-based monitoring and guidance for HIFU may bring a convenient solution for practical challenges associated with the use of magnetic-resonance imaging, such as economics, lack of portability, and material incompatibility.

\section{Acknowledgments}

This work was supported by the Biomedical Engineering Research Fund granted by Riverside Research Institute and a grant from the National Cancer Institute R44CA110137.

\section{References}

1. G. T. Clement, "Perspectives in clinical uses of high-intensity focused ultrasound," Ultrasonics 42, 1087-1093 (2004).

2. T. A. Leslie and J. E. Kennedy, "High-intensity focused ultrasound principles, current uses, and potential for the future," Ultrasound Quart. 22(4), 263-272 (2006).

3. K. Hynynen, O. Pomeroy, and D. N. Smith, "MR-imaging-guided focused ultrasound surgery of fibroadenomas in the breast: a feasibility study," Radiology 219, 176-185 (2001).

4. R. Mass-Moreno, C. A. Damianou, and N. T. Sanghvi, "Noninvasive temperature estimation in tissue via ultrasound echo-shifts. Part II. In vitro study," J. Acoust. Soc. Am. 100, 2522-2530 (1994). 
5. A. Anand and P. Kaczkowski, "Noninvasive measurement of local thermal diffusivity using backscattered ultrasound and focused ultrasound heating," Ultrasound Med. Biol. 34(9), 1449-1464 (2008).

6. A. A. Oraevsky, S. L. Jacques, R. O. Esenaliev, and F. K. Tittel, "Time-resolved optoacoustic imaging in layered biological tissues," in OSA Proc. on Advances in Optical Imaging and Photon Migration, Robert R. Alfano, Ed., Vol. 21, pp. 161-165 (1994).

7. A. A. Oraevsky and A. A. Karabutov, "Optoacoustic tomography," in chapter 34 Biomedical Photonics Handbook, T. Vo-Dinh, Ed., vol. PM125, pp. 34/1-34/34, CRC Press, Boca Raton, FL (2003).

8. S. A. Ermilov, T. Khamapirad, A. Conjusteau, R. Lacewell, K. Mehta, T. Miller, M. H. Leonard, and A. A. Oraevsky, "Laser optoacoustic imaging system for detection of breast cancer," J. Biomed. Opt. 14(2), 024007 (1-4) (2009).

9. R. O. Esenaliev, M. Motamedi, A. A. Karabutov, and A. A. Oraevsky, "Laser optoacoustic technique for real-time measurement of thermal damage in tissues," Proc. SPIE 3594, 98-109 (1999).

10. G. M. Spirou, I. A. Vitkin, B. C. Wilson, W. M. Whelan, P. M Henrichs, K. Mehta, T. Miller, A. Yee, J. Meador, and A. A. Oraevsky, "Development and testing of an optoacoustic imaging system for monitoring and guiding prostate cancer therapies," Proc. SPIE 5320, 44-56 (2004).

11. T. D. Khokhlova, I. M. Pelivanov, O. A. Sapozhnikov, V. S. Solomatin, and A. A. Karabutov, "Opto-acoustic diagnostics of the thermal action of high-intensity focused ultrasound on biological tissues: the possibility of its applications and model experiments," Quantum Electron. 36(12), 1097-1102 (2006).

12. K. V. Larin, I. V. Larin, and R. O. Esenaliev, "Monitoring of tissue coagulation during thermotherapy using optoacoustic technique," $J$ Phys. D: Appl. Phys. 38, 2645-2653 (2005).

13. A. A. Oraevsky, S. L. Jacques, and F. K. Tittel, "Determination of tissue optical properties by time-resolved detection of laser-induced stress waves," Proc. SPIE 1882, 86-101 (1993).

14. L. V. Wang, Ed. "Photoacoustic imaging and spectroscopy," Taylor and Francis Group, New York, 2009.

15. Y. Lao, D. Xing, S. Yang, and L. Xiang, "Noninvasive photoacoustic imaging of the developing vasculature during early tumor growth," Phys. Med. Biol. 53, 4203-4212 (2008).

16. G. Lungu, M. Li, X. Xie, L. Wang, and G. Stoica, "In vivo imaging and characterization of hypoxia-induced neovascularization and tumor invasion," Int. J. Oncol. 30, 40-54 (2007).

17. A. Oraevsky, E. Savateeva, S. Solomatin, A. Karabutov, V. Andreev, Z. Gatalica, T. Khamapirad, and P. Henrichs, "Optoacoustic imaging of blood for visualization and diagnostics of breast cancer," Proc. SPIE 4618, 81-94 (2002).

18. T. Khamapirad, P. Henrichs, K. Mehta, T. Miller, A. Yee, and A.
Oraevsky, "Diagnostic imaging of breast cancer with LOIS: clinical feasibility," Proc. SPIE 5697, 35-44 (2005).

19. X. Wang, Y. Pang, G. Ku, X. Xie, G. Stoica, and L. Wang, "Noninvasive laser-induced photoacoustic tomography for structural and functional in vivo imaging of the brain," Nature Biotechnol. 21, 803806 (2003)

20. S. Manohar, S. Vaartjes, J. van Hespen, J. Klaase, F. van den Engh, W. Steenbergen, and T. van Leeuwen, "Initial results of in vivo noninvasive cancer imaging in the human breast using near-infrared photoacoustics," Opt. Express 15(19), 12277-12285 (2007).

21. H. Brecht, R. Su, M. Fronheiser, S. Ermilov, A. Conjusteau, A. Liopo, M. Motamedi, and A. Oraevsky, "Optoacoustic 3-D whole body tomography: experiments in nude mice," Proc. SPIE $\mathbf{7 1 7 7}$ 71770E (2009)

22. J. P. Sedelaar, R. G. Aarnink, G. J. van Leenders, H. P. Beerlage, F. M. Debruyne, H. Wijkstra, and J. J. de La Rosette, "The application of three-dimensional contrast-enhanced ultrasound to measure volume of affected tissue after HIFU treatment for localized prostate cancer," Eur. Urol. 37(5), 559-568 (2000).

23. N. L. Bush, I. Rivens, G. R. Ter Harr, and J. C. Bamber, "Acoustic properties of lesions generated with an ultrasound therapy system," Ultrasound Med. Biol. 19(9), 789-801 (1993).

24. A. R. Funke, J. Aubry, M. Fink, A. Boccara, and E. Bossy, "Photoacoustic guidance of high-intensity focused ultrasound with selective optical contrasts and time-reversal," Appl. Phys. Lett. 94, 054102 (2009).

25. J. A. Copland, M. Eghtedari, V. L. Popov, N. Kotov, N. Mamedova, M. Motamedi, and A. A. Oraevsky, "Bioconjugated gold nanoparticles as a molecular-based contrast agent: implications for imaging of deep tumors using optoacoustic tomography," Molecular Imag. Biol. 6(5), 341-349 (2004).

26. S. Y. Emelianov, S. R. Aglyamov, J. Shah, S. Sethuraman, W. G. Scott, R. Schmitt, M. Motamedi, A. Karpiouk, and A. A. Oraevsky, "Combined ultrasound, optoacoustic, and elasticity imaging," Proc. SPIE 5320, 101-112 (2004).

27. J. J. Niederhauser, M. Jaeger, R. Lemor, P. Weber, and M. Frenz, "Combined ultrasound and optoacoustic system for real-time highcontrast vascular imaging in vivo," IEEE Trans. Med. Imaging 24(4), 436-440 (2005)

28. R. Esenaliev, A. Oraevsky, K. Larin, I. Larina, and M. Motamedi, "Real-time optoacoustic monitoring of temperature in tissues," Proc. SPIE 3601, 268-275 (1999).

29. A. A. Karabutov, E. V. Savateeva, N. B. Podymova, and A. A. Oraevsky, "Backward mode detection of laser-induced wide-band ultrasonic transients with optoacoustic transducer," J. Appl. Phys. 87(4), 2003-2014 (2000). 